Göteborg preprint

hep-th/0006073

June, 2000

\title{
D-branes, Cyclic Symmetry and Noncommutative Geometry
}

\author{
SUPRIYA KAR円 \\ Institute for Theoretical Physics \\ Göteborg University and Chalmers University of Technology \\ S-412 96 Göteborg, Sweden
}

(November 13, 2018)

\begin{abstract}
We investigate the open string modes, describing the world-volume of a D p-brane, for its cyclic symmetry in presence of a magnetic field. It is argued that the constant coordinate modes receive non-perturbative correction. We show that they introduce the notion of noncommutativity on the D p-brane world-volume and make it UV-renormalizable. An analogy between cyclic symmetry ( $\alpha^{\prime}$-corrections) and the noncommutative geometry $\left(\Theta_{A^{-}}\right.$-corrections $)$is presented to explain some of the unusual IR phenomena often noticed in a noncommutative theory.
\end{abstract}

\footnotetext{
${ }^{1}$ supriya@fy.chalmers.se
} 


\section{Introduction}

Noncommutative geometry has renewed its interest in a non-perturbative formulation [1], stimulated with the work [2] by Connes, Douglas and Schwarz. It is shown that M-theory in a constant three-form background is equivalent to a super Yang-Mills theory on a noncommutative torus. The interest has further gained momentum with the work [3] by Seiberg and Witten where an equivalence between Yang-Mills symmetry and a noncommutative gauge symmetry has been established. Since the theory includes gravity, one of the important problem is the consistency of a noncommutative theory or its renormalizability. Interestingly, a great deal of work describing loop-renormalizations in a noncommutative theory has been discussed in the current literatures [4]-[10]. It is known that a noncommutative description shares some common features with that of an open string theory [11, 12].

In this context, we shall present a comparative study between the stringy $\left(\alpha^{\prime}\right)$ and a noncommutative $\left(\Theta_{A}\right)$ corrections to an ordinary quantum field theory (QFT). Under either correction, the space is described by quantum operators though they possess very different geometries. The $\alpha^{\prime}$ correction are perturbative and introduces the notion of a string of finite length. On the other hand, we shall note that the $\Theta_{A}$ gives rise to the geometric corrections and also can be seen to introduce a minimum length scale in the theory. In fact, both the symmetries are intriguing to understand the ultra-violet (UV) and infra-red (IR) renormalizations in the respective theories.

Since a Dirichlet (D) p-brane [1] is defined at the boundary of an open string, it retains the cyclic symmetry on its world-volume. In presence of a magnetic field (i.e. an antisymmetric two form B-field), the D p-brane world-volume possesses a noncommutative symmetry. Then, the world-volume turns out to be an ordered space [3]. Thus, both the cyclic and noncommutative symmetry, independently, lead to an ordered space description on the D p-brane world-volume. In fact, it is the conjugate momentum on the world-volume of a $\mathrm{D}$ p-brane which receives correction due to B-field and introduces the notion of noncommutativity. As a result, the string coupling $g_{s}$ can be seen to be associated with the noncommutative parameter. With a simple analysis, we note that the geometric correction to the world-volume coordinates is nonperturbative nature. Interestingly, both $\alpha^{\prime}$ and $\Theta_{A}$ corrections can be seen to contribute identical phase factors to the interacting vertices (or amplitude). In addition, with $\alpha^{\prime}$-correction, a Tduality symmetry is known to relate $s$ and $t$-channel amplitudes though very different phenomena

\footnotetext{
${ }^{2}$ The phase can be seen to be symmetric with the cyclic ordering and is antisymmetric in case of the noncommutative symmetry. It is the antisymmetric phase, which turns out to be non-trivial where as the symmetric one does not contribute substantially with the on-shell condition. In fact, the latter is a requirement for the cyclic symmetry.
} 
known to occur in either channels [13]. Similarly, some of the divergences in an ordinary theory can be seen to be regularized with the geometric correction. Thus, it might be possible to explain some of the important phenomena in a noncommutative theory with the tools from the stringy corrections.

In this paper, we investigate the cyclic and noncommutative symmetries on the world-volume of a D p-brane in an open bosonic string theory. We consider a consistent Fourier mode expansion for the world-volume coordinates in presence of a magnetic field and obtain the equal-time commutation relation for the zero modes as well as for the non-zero modes. Then, the constant coordinate modes turn out to be noncommutative where as the non-zero momentum modes are modified due to the magnetic field. The latter observation is due to the fact that the world-volume metric receives correction in a noncommutative theory. The analysis for the zero modes suggests that the center of mass coordinates for a D p-brane become operators and are completely responsible for the noncommutative description. Then, the stringy analysis for the noncommutavity on the world-volume reduces precisely to that of a quantum field theory. We compute the phase factor for a set of scalar fields in a momentum space of the noncommutative quantum field theory (NQFT). We analyze the non-trivial phase contribution to exploit some of the divergence problems of an ordinary quantum field theory. We perform the analysis in openstring channel as well as its dual closed string channel and explain some of the IR phenomena observed [6] in a noncommutative theory.

We plan to present the paper as follows. In section 2, we briefly re-view some of the D p-brane features in an open string channel. We discuss the noncommutativity among the zero modes in section 3.1 and the compute for the non-local phase factor in 3.2. The field theoretic limit is discussed in section 3.3. The section 4 deals with the UV/IR connection, where in 4.1 we discuss the cyclic ordering on a $\mathrm{D}$ p-brane world-volume in presence of a magnetic field. We explain some of the IR-phenomena due to zero modes in sections 4.2 and 4.3. Finally, we conclude with some out-look in section 5 .

\section{Preliminaries}

\subsection{D p-brane world-volume and cyclic symmetry}

Let us begin with an infinite-strip world-sheet topology, $\mathcal{I}_{[0, \pi]} \times \mathcal{R}$, for an open string. Then the space-like coordinate, $0 \leq \sigma \leq \pi$, corresponds to the length of the string where as $\tau$ is time-like.

The mode expansion for the string coordinates, $X^{\mu}(\sigma, \tau)$, in term of its zero modes (coordinate: 
$x^{\mu}$ and momentum $p^{\mu}$ ) and non-zero modes ( momentum: $\alpha_{m}^{\mu}$ ) can be given by

$$
X^{\mu}(\sigma, \tau)=x^{\mu}+2 \alpha^{\prime} p^{\mu} \tau+i \sqrt{2 \alpha^{\prime}} \sum_{m \neq 0} \frac{1}{m} \alpha_{m}^{\mu} e^{-i m \tau} \cos m \sigma .
$$

Consider an arbitrary D p-brane in the open bosonic string theory. The D p-brane longitudinal coordinates can be defined at the open string boundaries 3, $\sigma=0$ and $\sigma=\pi$, with Neumann condition. In this picture, the world-volume coordinate fields $X^{i}$ are real and so its zero modes. Also the D p-brane non-zero modes $(m \neq 0)$ satisfy the hermiticity condition: $\alpha_{-m}^{i}=\left(\alpha_{m}^{i}\right)^{\dagger}$ which is evident from the mode expansion (11). The equal-time $(\tau)$ canonical commutation relation for the world-volume coordinates can be re-written from that of the open string:

$$
\left[X^{i}(\sigma, \tau), P^{j}\left(\sigma^{\prime}, \tau\right)\right]=i g^{i j} \delta\left(\sigma-\sigma^{\prime}\right),
$$

where $P^{i}=\left(2 \pi g_{s} \alpha^{\prime}\right)^{-1} \partial_{\tau} X^{i}$ represents the conjugate momenta and $\left(2 \pi g_{s} \alpha^{\prime}\right)$ signifies the D p-brane tension. $g_{i j}$ denotes the induced metric on the world-volume. For simplicity, we consider $g_{00}=-1$ and $g_{i j}=g \delta_{i j}$ for $i \neq 0$. Similarly, the commutation relation among the brane modes can be obtained at the string boundary $\partial \Sigma$. The only non-vanishing commutator turns out to be the one among its non-zero momentum modes:

$$
\left[\alpha_{m}^{i}, \alpha_{m^{\prime}}^{j}\right]=\left(2 \alpha^{\prime} m\right) g^{i j} \delta_{m+m^{\prime}}
$$

The commutation relation implies that the non-zero oscillator modes $(m \neq 0)$ can act as creation and anhilation operators, respectively, for $m<0$ and $m>0$ in the Fock space. A careful analysis of the non-zero modes towards its cyclic ordering (in $\tau$ ) among the vertex operators can be performed. For instance, the identity for two tachyon vertices with external momentum $k_{1}$ and $k_{2}$ turns out to be

$$
\mathcal{V}_{1}\left(k_{1}, \tau_{1}\right) \mathcal{V}_{2}\left(k_{2}, \tau_{2}\right)=\mathcal{V}_{2}\left(k_{2}, \tau_{2}\right) \mathcal{V}_{1}\left(k_{1}, \tau_{1}\right) \cdot \exp \left(2 i \pi \alpha^{\prime} k_{1} \cdot k_{2} \mathcal{E}\left(\tau_{1}-\tau_{2}\right)\right)
$$

where $\mathcal{E}(\tau)$ is a constant and is not well defined at $\tau=0$. Then for $n$-particles with external momentum $k_{n}$, the vertex operators $\mathcal{V}_{n}\left(k_{n}\right)$ can be shown to be associated with a phase factor:

$$
\mathcal{V}_{1}\left(k_{1}, \tau_{1}\right) \ldots \mathcal{V}_{n}\left(k_{n}, \tau_{n}\right) \longrightarrow \exp \left(2 i \pi \alpha^{\prime} \sum_{n<n^{\prime}} g^{i j} k_{n i} k_{n^{\prime} j} \mathcal{E}\left(\tau_{n}-\tau_{n^{\prime}}\right)\right) \mathcal{V}_{1}\left(k_{1}, \tau_{1}\right) \ldots \mathcal{V}_{n}\left(k_{n}, \tau_{n}\right)
$$

However, using the momentum conservation, the phase factor reduces to an identity which is a requirement of the cyclic symmetry and leads to an unitary description.

\footnotetext{
${ }^{3} X_{L}^{i}(0, \tau)$ and $X_{R}^{i}(\pi, \tau)$ for $i=(0,1,2, \ldots p-1)$ correspond respectively to the left and right ends of the open string. Since the left and right modules are commutant of each other, it is sufficient to consider a single module of the string for the analysis.

${ }^{4}$ Here after, the open string boundary is assumed to be implicit and we shall directly refer to a D p-brane.
} 


\subsection{Noncommutative description on the world-volume}

Now consider a magnetic field $\left(B_{0 i}=0\right)$ on the world-volume of a $\mathrm{D}$ p-brane. The magnetic field can be seen to introduce non-perturbative correction to the world-volume coordinates $X^{i} \rightarrow \hat{X}^{i}$ and makes it noncommutative.

Naturally, the magnetic field significantly modifies the the effective dynamics of a D p-brane.甲 The on-shell condition for the world-volume coordinates turns out to be:

$$
g_{i j} \partial_{\sigma} \hat{X}^{j}+\left.\bar{B}_{i j} \partial_{\tau} \hat{X}^{j}\right|_{\partial \Sigma}=0
$$

In this case, the equal-time commutation relation for the conjugate coordinates satisfies (2). However, the conjugate momenta receives correction due to the magnetic field. The momenta can be given by

$$
\hat{P}^{i}=\frac{1}{2 \pi g_{s} \alpha^{\prime}}\left[g^{i j} \partial_{\tau} \hat{X}_{j}+\bar{B}^{i j} \partial_{\sigma} \hat{X}_{j}\right] .
$$

In presence of a magnetic field, the equal-time commutation relation for the world-volume coordinates turns out to be

$$
\left[\hat{X}^{i}(\tau), \hat{X}^{j}(\tau)\right]= \pm i\left(2 \pi \alpha^{\prime}\right) \Theta_{A}^{i j}
$$

where $\Theta_{A}^{i j}$ is an antisymmetric matrix and is invertible. Explicitly!

$$
\Theta_{A}^{i j}=g_{s}\left(\frac{1}{g+\bar{B}} \bar{B} \frac{1}{g-\bar{B}}\right)^{i j} .
$$

In fact, it is the antisymmetric property of an induced field that gives rise to a noncommutative description on the $\mathrm{D}$ p-brane world-volume. The noncommutativity of the world-volume coordinates in presence of a magnetic field is a new phenomena and can be seen essentially due to its non-perturbative corrections. For instance, in absence of a magnetic field, the r.h.s. in the commutation relation (8) vanishes and implies an ordinary geometry. It can be checked that any perturbative correction (in $\Theta_{A}$ ) to the world-volume coordinates can not change an ordinary geometry to a noncommutative one.

\footnotetext{
${ }^{5}$ The dynamics can be obtained from the disk amplitude modulo closed string vertex with the appropriate boundary conditions. In an orthonormal moving frame [14], the action for a D p-brane simplifies drastically. The locally flat background fields, in the frame-work, facilitates the computation and leads to a general description for a D p-brane dynamics with symmetric and antisymmetric curvatures.

${ }^{6}$ For instance, the Dirac bracket for the open string coordinates at its boundary has been obtained in ref. 15. We follow the notions in ref. 16 .

${ }^{7}$ In an orthonormal frame, $\Theta_{A}^{i j}$ can be locally mapped to a constant matrix.
} 


\section{Brane modes in presence of a magnetic field}

\subsection{Zero modes noncommutativity}

Since a D p-brane is defined at the boundary of an open string, the world-volume can be described by the string modes. The Fourier mode expansion for the coordinate fields $X^{i}(\tau)$ can be obtained by drawing an analogy from the string mode expansion (1) which satisfies the modified boundary condition (6). It becomes:

$$
\hat{X}^{i}(\tau)=\hat{x}^{i}+\left(2 \alpha^{\prime}\right) \hat{p}^{i} \tau+\left.i \sqrt{2 \alpha^{\prime}} \sum_{m \neq 0} \frac{1}{m} \hat{\alpha}_{m}^{i} e^{-i m \tau} \cos m \sigma\right|_{\partial \Sigma} .
$$

Since the conjugate momenta (7) is modified in presence of a magnetic field, a careful analysis can be performed [14, 16] to see that the induced metric also receives correction. We recall [3] that the corrections are obtained at the open string boundary. As a result the effective metric in presence of a magnetic field turns out to be:

$$
g^{i j} \longrightarrow \Theta_{S}^{i j}=\left(\frac{1}{g+\bar{B}} g \frac{1}{g-\bar{B}}\right)^{i j}
$$

The canonical commutator (2) in presence of a magnetic field for the conjugate zero modes can be defined with the modified metric (11). It becomes

$$
\left[\hat{x}^{i}, \hat{p}^{j}\right]=i \Theta_{S}^{i j}
$$

Similarly, the commutation relation among the non-zero modes (3) in presence of a magnetic field can be rewritten as

$$
\left[\hat{\alpha}_{m}^{i}, \hat{\alpha}_{-m}^{j}\right]=\left(2 \alpha^{\prime} m\right) \Theta_{S}^{i j}
$$

However the equal-time canonical commutation relation (2) in presence of a magnetic field remains unaffected, since the corrections are incorporated in the momenta $\hat{P}^{i}$. For a D p-brane, the non-zero modes are constrained at the open string boundary to yield $\hat{P}^{i}=g_{s}^{-1} \hat{p}^{i}$. The commutator can be checked for the brane modes and becomes:

$$
\left[\hat{X}^{i}(\sigma, \tau), \hat{P}^{j}\left(\sigma^{\prime}, \tau\right)\right]=i g^{i j} \delta\left(\sigma-\sigma^{\prime}\right)
$$

where $\delta\left(\sigma-\sigma^{\prime}\right)=\pi^{-1}\left(1+\sum_{m \neq o} \cos m \sigma \cos m \sigma^{\prime}\right)$ defines at the string boundary. The analysis in presence of a magnetic field (14) re-confirms the world-volume commutation relation obtained in eq.(8).

\footnotetext{
${ }^{8}$ In general, $\delta\left(\sigma-\sigma^{\prime}\right)$ can be seen to be constrained by the oscillator modes, which allow $\sigma$ to take one value in bulk apart from its boundary values.
} 
Now the commutatation relation (8) can be re-written, independently, in terms of its zero and non-zero modes (10). Then the equal-time commutator reduces to the one among its zero modes $\hat{x}^{i}$. It can be given by

$$
\begin{array}{rlrl} 
& & {\left[\hat{x}^{i}, \hat{x}^{j}\right]= \pm i\left(2 \pi \alpha^{\prime}\right) \Theta_{A}^{i j}} \\
\text { and } \quad & {\left[\hat{p}^{i}, \hat{p}^{j}\right]=0 .}
\end{array}
$$

The non-vanishing of the commutation relation for the constant coordinate modes can be understood in terms of its non-perturbative correction. Since a magnetic field defines a noncommutative world-volume (8), it is the constant coordinate modes, $x^{i}$, which inherit the effective noncommutativity (15). The constant (canonical) momentum modes commute in this channel as they do not receive any correction.

On the other hand, the non-zero modes on the world-volume are modified in presence of a magnetic field (13). They are redundant to a noncommutative description though their role is vital towards an UV-renormalization in an ordinary theory.

\subsection{Non-local phase factor}

In this section, we compute the phase factor associated with the constant coordinate modes in a noncommutative description. A Weyl ordering prescription can be utilized to map an ordinary scalar field $\phi(x)$ to $\Phi(\hat{x})$ in an ordered space. Since the noncommutative world-volume is defined with $\left(\hat{x}^{i}-x^{i}\right) \neq 0$, a scalar field can be given by

$$
\Phi(\hat{x})=\frac{1}{(2 \pi)^{p}} \int d^{p} x d^{p} \hat{p} e^{i \hat{p}_{i} \cdot\left(\hat{x}^{i}-x^{i}\right)} \phi(x),
$$

where a note on the order of the homomorphic map $\phi(x) \rightarrow \phi(\hat{p}) \rightarrow \Phi(\hat{x})$ is implicit in eq. (16). Then, a point-wise multiplication is replaced by the point-less (noncommutative) $\star$-product. The scalar interaction term for $n$-number of particles can be given in its momentum space:

$$
\begin{aligned}
\Phi(\hat{x}) \star \ldots \star \Phi(\hat{x})=\frac{1}{(2 \pi)^{p / 2}} \int d^{p} \hat{p}_{1} \ldots d^{\hat{p}} p_{n} & \\
& \cdot e^{i p_{1} \cdot \hat{x}} \phi\left(\hat{p}_{1}\right) \ldots e^{i \hat{p}_{n} \cdot \hat{x}} \phi\left(\hat{p}_{n}\right) .
\end{aligned}
$$

A simplification of the integrand can be performed with the help of the commutation relation (15). Then the $n$-particle scalar interaction (17) reduces to:

$$
\begin{gathered}
{[\Phi(\hat{x})]^{n}=\frac{1}{(2 \pi)^{p / 2}} \int d^{p} \hat{p}_{1} \ldots d^{p} \hat{p}_{n} \cdot \exp \left(i \sum_{n} \hat{p}_{n} \cdot \hat{x} \pm i \sum_{n<n^{\prime}} \hat{p}_{n i} \Theta_{A}^{i j} \hat{p}_{n^{\prime} j}\right)} \\
{\left[\phi\left(\hat{p}_{1}\right) \ldots \phi\left(\hat{p}_{n}\right)\right] .}
\end{gathered}
$$


The integrand confirms that the $n$-particle interaction vertex is associated with a phase factor in a noncommutative description. It implies that the internal momentum $\hat{p}$ plays significant role in a noncommutative theory. The momentum conservation condition further simplifies the $n$-particle interactions and the integrand correspond precisely to a vertex $\mathcal{V}_{\phi}\left(\hat{p}_{1}, \ldots, \hat{p}_{n}\right)$ in the momentum space. It is given by

$$
\mathcal{V}_{n \phi}\left(\hat{p}_{1}, \ldots, \hat{p}_{n}\right)=\delta^{(p)}\left(\sum_{n} \hat{p}_{n}\right) \exp \left( \pm i \sum_{n<n^{\prime}} \hat{p}_{n i} \Theta_{A}^{i j} \hat{p}_{n^{\prime} j}\right) \cdot\left[\phi\left(\hat{p}_{1}\right) \ldots \phi\left(\hat{p}_{n}\right)\right] .
$$

It shows that the (scalar) interaction vertex on a noncommutative world-volume is associated with a non-trivial phase factor in addition to the one obtained (5) due to the cyclic property. Thus a non-local quantum field theory may be expressed in terms of a phase factor due to the constant modes and an ordinary quantum field theory, which can be seen to be UV-renormalized. Then the scattering amplitude in a noncommutative theory can be given by a combination of its oscillatory phase defined with the internal momentum $p$ and an UV renormalized QFT for the interacting particles with external momentum $k$. Schematically:

$$
\mathcal{A}\left(k_{n}, \hat{p}_{n} ; k_{n^{\prime}}, \hat{p}_{n^{\prime}}\right)_{N Q F T} \equiv \exp \left( \pm i \sum_{n<n^{\prime}} \hat{p}_{n i} \Theta_{A}^{i j} \hat{p}_{n^{\prime} j}\right) \cdot \overline{\mathcal{A}}\left(k_{n}, k_{n^{\prime}}\right)_{Q F T}
$$

where an appropriate momentum conservation holds at the interacting vertices. The expression (20) is supported by the fact that the zero modes describe the ground state of a closed string and are massless. The wave function for a D p-brane in its ground state can be seen to be degenerate though energy level is not. Indeed, the multi-valued ground state wave function gives rise to a non-local description [16]. Then the task reduces to the computation of the non-local phase factor (20). In fact, the phase dominates over the usual behavior of momentum in the theory and softens the loop divergences.

Finally, we conclude the section with a note that the zero modes on the world-volume receive non-perturbative correction in presence of a magnetic field. They describe a noncommutative theory and contains its ordinary QFT counterpart. The fact lead to a conjecture that a noncommutative theory is renormalizable if its ordinary counterpart does.

\subsection{Field theoretic limit $\left(\alpha^{\prime} \rightarrow 0\right)$}

In the previous section, we noticed that a $\mathrm{D}$ p-brane center of mass coordinates decide the nature of its world-volume geometry. In presence of a magnetic field, the constant modes $\hat{x}^{i}$ receive geometric corrections (in $\Theta_{A}$ ) and the world-volume turns out to be noncommutative. On the 
other hand, the non-zero modes are associated with the stringy corrections (in $\alpha^{\prime}$ ) and do not play any role towards its world-volume geometric corrections. Thus, these two corrections are independent of each other.

In a field theoretic limit, $\alpha^{\prime} \rightarrow 0$, the non-zero modes are dropped out and the worldvolume retains its noncommutative description. A priori, the UV-renormalization seems to be not guaranteed in the field theoretic frame-work. However a close note on the world-volume noncommutative description (8), yields an uncertainty relation:

$$
\Delta \hat{x}^{i} \cdot \Delta \hat{x}^{j} \geq \frac{1}{2}\left|\Theta_{A}^{i j}\right|
$$

It confirms a finite value to the, naive, short-distance divergence by assigning a critical cutoff value $\left(\Delta \hat{x}^{i}\right)_{\text {crit }} \neq 0$ for $i \neq j$. As a result, a minimum length scale is introduced into a noncommutative theory which takes care the UV-divergences. For instance, keeping a particle position $\left(\hat{x}^{i}\right)$ fixed, the uncertainty relation (21) would lead to non-local description in the $(i \neq j)$-directions. Since in absence of either $\left(\alpha^{\prime}\right.$ or $\left.\Theta_{A}\right)$ corrections, the space contains UVdivergences, these two parameters seem to play an identical role towards the short-distance features of a theory. Geometrically, $\alpha^{\prime}$ and $\Theta_{A}$ show their differences, nevertheless their UVfeatures can be seen to be identical in section 4 . For instance, the $\alpha^{\prime}$-correction introduces infinite number of particles in the spectrum and defines a string of length $\sqrt{2 \alpha^{\prime}}$ where as the $\Theta_{A}$-correction gives rise to a large density of particles in the ground state (zero modes) and makes it non-local. As explained, the latter phenomena introduces the notion of a noncommutative geometry on a D p-brane world-volume.

\section{$4 \quad$ UV and IR phenomena}

\subsection{Cyclic symmetry in presence of a magnetic field}

We begin this section by considering a two point correlator [14] for the open string vertex operators evaluated at the string boundary. Effectively, it describes a propagator on a D pbrane world-volume. The Neumann propagator in a matrix notation can be written as

$$
\left\langle\hat{X}^{i}(\tau) \hat{X}^{j}\left(\tau^{\prime}\right)\right\rangle=4 \pi \alpha^{\prime} \Theta_{S}^{i j} \ln \left(\tau-\tau^{\prime}\right)-i \pi \alpha^{\prime} \Theta_{A}^{i j} \mathcal{E}\left(\tau-\tau^{\prime}\right)
$$

where $\mathcal{E}(\tau)$ introduces the notion of $\tau$-ordered space on the string world-sheet. In the alternate picture, the noncommutative description on the world-volume (8) can be re-written in terms $\tau$ ordering at the string boundary. An appropriate combination of the propagators in the ordered

\footnotetext{
${ }^{9}$ It is associated with an antisymmetric exchange of $i \leftrightarrow j$ indices
} 
space for the world-volume operators $\hat{X}^{i}(\tau)$ turns out to be identical. It can be given by

$$
T\left[\hat{X}^{i}(\tau) \hat{X}^{j}\left(\tau^{\prime}\right)-\hat{X}^{i}\left(\tau^{\prime}\right) \hat{X}^{j}(\tau)\right]=i\left(2 \pi \alpha^{\prime}\right)\left(2 \Theta_{S}^{i j}-\Theta_{A}^{i j}\right) \mathcal{E}\left(\tau-\tau^{\prime}\right) .
$$

Now, interchanging the notion of time-ordering with the antisymmetric exchange of brane coordinates, one can define the limit $\tau \rightarrow \tau^{\prime}$. Then, with a little subtleties, one can obtain the equal-time world-volume commutation relations (8) from the time-ordered combination (23). The analysis implies that an ordered space in presence of a B-field can be equivalently described by a noncommutative space. The antisymmetrization among the world-volume operators $\hat{X}^{i}(\tau)$ naturally forbids the non-zero modes commutation relation (13). The analysis further assures the relevance of zero coordinate modes $\hat{x}^{i}$ towards a noncommutative geometry. A priori the result may appear surprising, since it is the strong magnetic field (large B) limit where the zero modes play a leading role towards the world-volume geometry. However it works, since the usual role of non-zero modes 10 is auxiliary in a noncommutative description. II It implies that the analysis for a large B-field may be generalized to any $B \neq 0$. In particular, the large $\mathrm{B}$ limit is an important domain where the theory turns out to be topological and holographic idea can be studied there.

\subsection{Origin of virtual particles and IR-singularities}

Until now, we have been discussing the D p-brane modes in an open string channel (t-channel). We learned that in a noncommutative description, the uncertainty relation (21), among the constant coordinate modes $\hat{x}^{i}$, takes care the otherwise short-distance divergences. In addition, these modes are non-propagating ones which is evident from their phase contribution (19). In the open string channel, the constant modes (10) can be easily identified with that of a closed string and are massless. Since the constant momentum modes $p^{i}$ commute (15), the closed string zero modes do not possess a lower cut-off limit in its internal momentum. As a result, the loop integral for the zero modes exhibit the usual IR singularities on the D p-brane world-volume.

To be explicit, consider $n$ (massive) particle interaction vertex momenta $\left(k_{1}, \ldots, k_{n^{\prime}}, \ldots, k_{n}\right)$ in a noncommutative theory. In a point-splitting prescription,

\footnotetext{
${ }^{10}$ They play a crucial role towards the UV-renormalization in an ordinary theory.

${ }^{11}$ Alternately, the noncommutative description defined by the equal-time commutation relation (8) can be checked for the brane modes (10). Formally the modified metric $\Theta_{S}^{i j}$ on the r.h.s. of the commutator can be seen to be associated with a divergent piece $\left(-2 \alpha^{\prime} \ln m\right)$ due to the infinite tower of non-zero modes. In an ordinary theory the $\zeta$-function regularization takes care of the divergence. On the other hand, a noncommutative description does not permit any symmetric (in $i \leftrightarrow j$ ) term on the r.h.s. of its world-volume commutation relation (8) and hence forbids the otherwise divergent piece.

${ }^{12}$ In case of scalar particles, the interaction vertex has been obtained in eq.(19).
} 
the interaction vertex $\mathcal{V}_{n}$ splits into two vertices, each consisting of $n^{\prime}$ and $\left(n-n^{\prime}\right)$ interacting particles, and are connected by the matrix correlator

$$
\left\langle\hat{\phi}_{0}^{i}(-p) \hat{\phi}_{0}^{j}(p)\right\rangle= \pm\left(\pi \alpha^{\prime}\right) i \Theta_{A}^{i j}
$$

Here $\hat{\phi}_{0}^{i}$ denote the closed string zero modes and represent virtual particles in the momentum space. The $n$-particle vertex can be illustrated by a point-splitting prescription:

$$
\mathcal{V}\left(k_{1}, \ldots, k_{n^{\prime}}, k_{n^{\prime}+1}, \ldots, k_{n}\right) \rightarrow \mathcal{V}_{1 i}\left(k_{1}, \ldots, k_{n^{\prime}}\right)\left(i \Theta_{A}^{i j}\right) \mathcal{V}_{2 j}\left(k_{n^{\prime}+1}, \ldots, k_{n}\right)
$$

where the momentum conservation and the cyclic symmetry are preserved at both the vertices. Intuitively, the point-splitting prescription is identical to the stringy phenomenon involving different channels. Alternately, the point-splitting prescription (25) can be described by two independent vertices with momentum conservation condition at each of them:

$$
\tilde{\mathcal{V}}_{1}\left(k_{1}, \ldots, k_{n^{\prime}},-p\right) \cdot \tilde{\mathcal{V}}_{2}\left(p, k_{n^{\prime}+1}, \ldots, k_{n}\right)
$$

For scalar particles, the vertices can be expressed explicitly:

$$
\begin{aligned}
& \tilde{\mathcal{V}}_{1 \phi}=\delta^{(p)}\left(k_{1}+\ldots+k_{n^{\prime}}-p\right) \cdot \exp \left( \pm i p_{i} \Theta_{A}^{i j} p_{j}\right)\left[\phi\left(k_{1}\right) \ldots \phi\left(k_{n^{\prime}}\right) \hat{\phi}_{0}(-p)\right] \\
& \text { and } \quad \tilde{\mathcal{V}}_{2 \phi}=\delta^{(p)}\left(k_{n^{\prime}+1}+\ldots+k_{n}+p\right) \cdot \exp \left( \pm i p_{i} \Theta_{A}^{i j} p_{j}\right)\left[\phi\left(k_{n^{\prime}+1}\right) \ldots \phi\left(k_{n}\right) \hat{\phi}_{0}(p)\right] .
\end{aligned}
$$

The momentum conservation at each vertex yields:

$$
\begin{aligned}
\tilde{\mathcal{V}}_{1 \phi} & =\exp \left( \pm i k \cdot p_{n c}\right)\left[\phi\left(k_{1}\right) \ldots \phi\left(k_{n^{\prime}}\right) \hat{\phi}_{0}(-p)\right] \\
\text { and } \quad \tilde{\mathcal{V}}_{2 \phi} & =\exp \left(\mp i k^{\prime} \cdot p_{n c}\right)\left[\phi\left(k_{n^{\prime}+1}\right) \ldots \phi\left(k_{n}\right) \hat{\phi}_{0}(p)\right],
\end{aligned}
$$

where $p_{n c}^{i}=\Theta_{A}^{i j} p_{j}$ defines a noncommutative momenta. There $k$ and $k^{\prime}$ denote the total momentum due to the external particles, respectively, with $\tilde{\mathcal{V}}_{1 \phi}$ and $\tilde{\mathcal{V}}_{2 \phi}$. Thus in a noncommutative description, the $n$-particle vertex can be described by two independent vertices with $\left(n^{\prime}+1\right)$ and $\left(n-n^{\prime}+1\right)$ number of interacting particles. The additional particles at both the vertices are essentially the closed string zero modes and give rise to the IR poles in the amplitude even for the massive external particles. The phenomenon has been addressed [6]-[9] explicitly for the massive scalar $\Phi^{4}(\hat{x})$-interaction in four dimensions. In fact, the t-channel analysis leads to a non-planar Feynman graphs where the oscillatory phase (20) dominates and softens the otherwise divergences. The limiting value to the effective momentum, in t-channel, turns out to be: $\Lambda_{t} \equiv p_{n c}$. In the limit $\Theta_{A} \rightarrow 0$, the effective cut-off $\Lambda_{t} \rightarrow \infty$ and corresponds to the one in an ordinary theory. However, the cut-off $\Lambda_{t}$ remains finite for the non-planar loop graphs. 


\subsection{Effective IR cut-off}

Under a T-duality symmetry, the one-loop amplitude in a t-channel can be transformed to a tree amplitude in closed string theory (s-channel). In absence of a magnetic field, the Neumann matrix propagator (22) becomes diagonal and contains a short-distance loop divergence in the limit $\tau \rightarrow \tau^{\prime}$. It corresponds to an UV-divergence in the t-channel and can be regulated by defining a limiting value in a standard prescription. The UV cut-off value is known to be interpreted as the IR limit in its dual (s-) channel. Thus in a string frame-work, very different phenomena can be seen to occur in different channels. A close look in a noncommutative theory reveals that some phenomena resemble to that of strings.

In presence of a magnetic field, the Neumann matrix (22) contains off-diagonal propagators in addition to the usual diagonal ones. In the limit $\tau \rightarrow \tau^{\prime}$, the underlying world-sheet theory contains a loop diagram. However, the zero modes describing the noncommutative geometry on the world-volume are free from loop divergences and the matrix propagator can be seen to be a constant: $\left\langle\hat{x}^{i} \hat{x}^{j}\right\rangle= \pm i \pi \alpha^{\prime} \Theta_{A}^{i j}$. Interestingly, the propagator for the zero modes can be illustrated by the non-planar Feynman graph 17.

Since a duality transformation can be seen to exchange the winding modes with the momentum ones, the world-sheet coordinates $(\sigma \leftrightarrow \tau)$ interchange their roles. In addition to it, the Dirichlet boundary conditions are exchanged with the Neumann. Under a duality, the D $\tilde{p}$-brane world-volume does not possess any magnetic field. On the other hand, the magnetic field lies in the transverse space to the brane. Thus in a closed string channel, the commutation relation among the constant modes turns out to be

$$
\begin{aligned}
& {\left[\tilde{x}^{i}, \tilde{x}^{j}\right]=0 } \\
\text { and } \quad & {\left[\tilde{p}^{i}, \tilde{p}^{j}\right]= \pm i\left(2 \pi \alpha^{\prime}\right) \Theta_{A}^{i j} . }
\end{aligned}
$$

The commutator for the zero momentum modes implies an uncertainty relation among them. Thus the zero momentum mode $\tilde{p}$ is bounded from below by the matrix parameter $\Theta_{A}^{i j}$. In this case, though the closed string zero modes are massless, the loop integral turns out to be free from any IR singularities. The IR cut-off value for the internal momentum $\tilde{p}$, in s-channel, turns

out to be: $\Lambda_{s}=1 / \sqrt{\left(\pi \alpha^{\prime}\right) \Theta_{A}}$, where $\Theta_{A}$ denotes a typical eigen-value for the matrix $\left(\Theta_{A}\right)_{i j}$. The limit $\Theta_{A} \rightarrow 0$ corresponds to planar Feynman graphs and obviously associated with the poles. Since the resulting amplitude in a scattering phenomena is obtained by summing over its channel amplitudes, it implies that a noncommutative theory always contain an ordinary counterpart which is in agreement with the expression (20). 


\section{Discussions}

We have presented some similarities between a noncommutative theory and an open string theory in a non-perturbative frame-work of a D p-brane. We learned that the zero modes in the theory are completely responsible for a noncommutative description on a D p-brane world-volume. Since in a field theoretic limit $\left(\alpha^{\prime} \rightarrow 0\right)$, the non-zero modes decouple, the world-volume of a $\mathrm{D}$ p-brane precisely describes a NQFT. This, in fact, gives rise to a non-local QFT. Thus the non-locality is essentially due to the zero modes in the theory. Since the zero modes are non-propagating fields, their contribution is expressed as a non-trivial phase factor in a momentum space. The phase obtained is defined with the internal momenta of the zero modes. In a scattering phenomena (i.e. with external momenta of interacting particles) the momentum conservation allows an interplay between the internal and external momenta and redefines the phase. The IR-domain was analyzed to conclude that the virtual particles correspond to the closed string zero modes and play a significant role in a noncommutative theory.

On the other hand, the non-zero modes are associated with the $\alpha^{\prime}$ perturbative corrections. It was argued that the $\Theta_{A}$ and $\alpha^{\prime}$ play an identical role towards the renormalizations. In other words, the role of zero modes in a noncommutative theory is identical to that of the non-zero modes in a string theory. Both the modes satisfy identical commutation relations. A pointsplitting prescription in the former, apparently seems to yield scattering processes identical to that in the T-duals in string theory. In addition, a noncommutative description correspond to a large number of particles (which is transparent in the large B limit) in the spectrum similar to that of string. A priori, there seems to be an ambiguity, at this level, since the string spectrum consists of massive particles where as the zero modes are mass-less. However, the ambiguity may be resolved by generalizing the large $B$ limit to any $B \neq 0$, where presumably the gravity would supplement for mass to the zero modes. It is in agreement with the fact of an IR cut-off for the zero modes in the theory. In the context it remains to understand, if holography has a role to play in a noncommutative theory.

\section{Acknowledgments}

I am grateful to the members of the string group, in the Institute, for their helpful comments during an informal presentation of this work at its early stage. The work is supported by the Swedish Natural Science Research Council. 


\section{References}

[1] J. Polchinski, Phys. Rev. Lett. 75 (1995) 4724 (hep-th/9510017).

[2] A. Connes, M.R. Douglas and A. Schwarz, J.H.E.P. 2 (1998) 003 (hep-th/9711162).

[3] N. Seiberg and E. Witten, J.H.E.P. 9908 (1999) (hep-th/9908142).

[4] C.P. Martin and D. Sanchez-Ruiz, Phys. Rev. Lett.83 (1999) 476 (hep-th/9903077).

[5] M. M. Sheikh-Jabbari, J.H.E.P.06 (1999) 015 (hep-th/9903107).

[6] S. Minwalla, M. Van Raamsdonk and N. Seiberg, J.H.E.P.02 (2000) 020 (hep-th/9912072);

M. Van Raamsdonk and N. Seiberg, J.H.E.P.0003 (2000) 035 (hep-th/0002186).

[7] I. Ya. Aref'eva, D.M. Belov and A.S. Koshelev, Phys. Lett. B476 (2000) 431 (hep-th/9912075).

[8] M. Hayakawa, Phys. Lett. B478 (2000) 394 (hep-th/9912094); hep-th/9912167.

[9] A. Rajaraman and M. Rozali, J.H.E.P.0004 (2000) 033 (hep-th/0003227).

[10] A. Armoni, hep-th/0005208.

[11] E. Witten, Nucl. Phys. B268 (1986) 253.

[12] D.J. Gross and N.A. Nekrasov, hep-th/0005204.

[13] M.B. Green, J.H. Schwarz and E. Witten, "Superstring theory" Volume I, Cambridge Univ.press (1987).

[14] S. Kar, Nucl. Phys. B554 (1999) 163 (hep-th/9812230); hep-th/9907117.

[15] C.-S. Chu and P.-M. Ho, Nucl. Phys. B550 (1999) 151 (hep-th/9812219); Nucl. Phys. B568 (2000) 447 (hep-th/9906192).

[16] S. Kar, Nucl. Phys. B577 (2000) 171 hep-th/9911251).

[17] T. Filk, Phys. Lett. B376 (1996) 53. 